\title{
Perihematomal Diffusion Restriction in Intracerebral Hemorrhage Depends on Hematoma Volume, But Does Not Predict Outcome
}

\author{
Sebastian Stösser ${ }^{\mathrm{a}} \quad$ Hermann Neugebauer $^{\mathrm{a}}$ Katharina Althaus ${ }^{\mathrm{a}}$ \\ Albert C. Ludolph ${ }^{a}$ Jan Kassubek ${ }^{a}$ Michael Schocke ${ }^{b}$ \\ ${ }^{a}$ Department of Neurology, University of Ulm, and ${ }^{\mathrm{b}}$ Department of Neuroradiology, University and Rehabilitation \\ Hospital Ulm, Ulm, Germany
}

\section{Key Words}

Intracerebral hemorrhage - Magnetic resonance imaging ·

Cerebral edema $\cdot$ Blood pressure

\begin{abstract}
Background: Perihematomal diffusion restriction (PDR) is a frequent finding in primary intracerebral hemorrhage $(\mathrm{ICH})$ on diffusion-weighted MRI. Its frequency, associated clinical and imaging findings and impact on clinical outcome are not well understood. Methods: This is a retrospective single-center analysis of 172 patients with primary ICH who received $\mathrm{MRI}$ within $24 \mathrm{~h}$ from symptom onset. PDR was defined as a reduction of apparent diffusion coefficient below $550 \times 10^{-6}$ $\mathrm{mm}^{2} / \mathrm{s}$. Multivariate regression analyses were used to assess independent imaging and clinical predictors of PDR. Clinical outcome was assessed using the modified Rankin scale (mRS) at discharge. Results: PDR was present in 88 patients (51.2\%). Median PDR volume was $1.1 \mathrm{ml}$ (interquartile range 0.2-4.2). Multivariate analyses identified hematoma volume as the key independent predictor of PDR. The volume of perihematomal edema, lobar hematoma location and low diastolic blood pressure at admission were further predictors. Although the occurrence of PDR correlated with in-hospital mortality ( 75.0 vs. $43.4 \%, p<0.001)$ and moderately severe to severe disability or death at discharge ( $m R S \geq 4 ; 56.4$ vs.
\end{abstract}

$27.8 \%, \mathrm{p}=0.002)$, PDR was not an independent predictor of clinical outcome. In contrast, hematoma volume, ventricular extension of hemorrhage and higher age independently predicted an adverse clinical outcome. Conclusions: PDR is common after primary ICH within $24 \mathrm{~h}$ of symptom onset. Hematoma volume was identified as the key predictor of PDR. Although PDR was associated with mortality and severe disability, this effect was confounded by established risk factors. These results do not support a role of early PDR as prognostic factor after $\mathrm{ICH}$ independent of hematoma volume.

(c) 2016 S. Karger AG, Basel

\section{Introduction}

Spontaneous intracerebral hemorrhage (ICH) is associated with high mortality and poor outcome [1]. Important prognostic factors are hematoma volume and location, ventricular extension and patient age $[2,3]$. Further, perihematomal edema (PHE) independently predicts clinical outcome [4-7]. Using diffusion-weighted imaging (DWI)-MRI, PHE can be differentiated into areas of increased or reduced water diffusion, which may represent vasogenic or cytotoxic edema, respectively [8]. Previous studies observed areas of restricted diffusion within the perihematomal zone (perihematomal diffu-

\section{KARGER}

E-Mail karger@karger.com

www.karger.com/ced
(C) 2016 S. Karger AG, Basel

1015-9770/16/0424-0280\$39.50/0
Sebastian Stösser, MD

Department of Neurology

University of Ulm, Oberer Eselsberg 45 DE-89081 Ulm (Germany)

E-Mail sebastian.stoesser@uni-ulm.de 
sion restriction, $\mathrm{PDR}$ ) in up to $70 \%$ of patients with $\mathrm{ICH}$, which was potentially associated with an unfavorable clinical outcome [9-12]. These studies were, however, limited by small sample sizes $(\mathrm{n}<40)$ and yielded contradictory results. A recent study investigated the properties of PDR in 117 patients with ICH who underwent MRI within 14 days after symptom onset [13]. This study observed PDR in $32.4 \%$ of their patients and identified hematoma volume as its sole independent predictor. Unfortunately, the study did not report on outcome data in relation to PDR.

The purpose of this study was to investigate properties and associated factors of PDR in a sizeable sample of patients with primary ICH who had received an MRI scan within $24 \mathrm{~h}$ of symptom onset. Further, we evaluated the prognostic value of early PDR for clinical outcome in these patients.

\section{Methods}

\section{Patients}

All patients with a diagnosis of ICH who were admitted to the Department of Neurology, University Hospital of Ulm, Germany, between January 2009 and September 2014 were retrospectively identified by diagnostic code. MRI was the principal neuroimaging modality in all stroke patients including hemorrhagic stroke. Patients who received an MRI scan immediately after admission and within $24 \mathrm{~h}$ after symptom onset were included in the study. Exclusion criteria were ICH due to vascular malformations, structural lesions, trauma, ruptured aneurysm, cerebral venous thrombosis, hemorrhagic transformation of an ischemic infarct or sub- and epidural hematoma. Further, patients with MRI scans confounded by motion or other artefacts were excluded. The study protocol was approved by the local Ethics Committee of the University of Ulm, Germany.

\section{Clinical and Laboratory Data}

Clinical and laboratory data at admission (pre-treatment) and medical history were collected by chart review. The clinical outcome was determined by chart review and retrospectively evaluated using the modified Rankin scale (mRS) [14].

\section{Imaging Studies}

All patients were imaged using a clinical 1.5 tesla MR scanner (Magnetom TIM Symphony, Siemens Erlangen, Germany) equipped with a 12-channel head coil. Transversal DWI was included in all studies with diffusion-sensitizing gradients in at least 3 orthogonal directions and $b$ values of 0 and $1,000 \mathrm{~mm}^{2} / \mathrm{s}$. Most studies additionally included transversal gradient recalled echo and T2-/T1-weighted sequences as well as coronal fluid attenuated inversion recovery (FLAIR) sequences. Imaging analysis was conducted using an in-house PACS system (Centricity PACS-IW, version 3.7.3.9078, General Electrics Healthcare, Milwaukee, USA) as well as the Fiji distribution of ImageJ (version 1.49; Wayne Rasband, National Institutes of Health) [15].

Diffusion Restriction in Intracerebral Hemorrhage
Hematoma and PHE were identified on baseline DWI images $(b=0)$ and manually outlined on each slice. Areas of restricted diffusion within PHE (hyperintense signal on DWI images $(b=$ $1,000)$ and hypointense signal on apparent diffusion coefficient (ADC) images were identified using a semi-automated thresholding technique with $\mathrm{ADC}<550 \times 10^{-6} \mathrm{~mm}^{2} / \mathrm{s}$ as a threshold. This threshold represents a $30 \%$ reduction of ADC compared to normal white matter, which is indicative of severely restricted diffusion as reached after prolonged ischemia [16-18]. This threshold has previously been used to assess PDR in ICH $[10,13]$. The ADC of a contralateral mirror ROI was measured as well. If no diffusion restriction could be identified within PHE, a representative ROI measurement was taken within PHE. The location of PDR in relation to the ventricular system was visually identified after manually dividing the hematoma in half along the anterior-posterior axis. The location of PDR in the superior or inferior half of axial DWI images was visually identified.

Volumes of hematoma, PDR and total lesion (hematoma and PHE) were calculated using a volumetric approach. PHE volume was determined by subtracting hematoma volume from total lesion volume $[6,19]$. Ventricular extension was identified using DWI, gradient recalled echo and FLAIR images. Small vessel disease was rated on FLAIR, T2-weighted images or B0 images according to the Fazekas scale [20]. The modified Boston criteria were used to assess possible/probable cerebral amyloid angiopathy (CAA) on MRI scans [21, 22]. Transversal gradient recalled echo sequences were examined for cortical superficial siderosis and cortical microbleeds. If cortical superficial siderosis and/or multiple cortical microbleeds as well as a single lobar hemorrhage were present, patients were classified as 'probable CAA'.

\section{Statistical Analysis}

Statistical analyses were performed using the Statistical Package for Social Sciences version 21.0.0.0 (IBM SPSS Statistics, Armonk, N.Y., USA). Differences in metric data between groups were assessed using Mann-Whitney $U$ tests. Differences in frequencies between groups were assessed using Pearson chi-square tests. Regression analysis was conducted using multivariate linear, logistic and ordinal models where appropriate. Power calculation were performed using G*Power version 3.1.9.2 (Franz Faul, University of Kiel, Germany) [23]. All tests were 2-tailed. Statistical significance was determined at an a level of 0.05 . As this was a retrospective exploratory study, no correction of a levels for multiple testing was used.

\section{Results}

A total of 527 patients were identified. Patients with ICH due to the following etiologies were excluded from the analysis: hemorrhagic transformation (including petechial hemorrhage) of ischemic infarct with or without thrombolytic therapy $(\mathrm{n}=213)$, metastatic/neoplastic lesions $(n=34)$, vascular malformations $(n=14)$, ruptured aneurysms $(n=10)$, cerebral venous thrombosis $(n=6)$, trauma $(\mathrm{n}=5)$ and subdural hematoma $(\mathrm{n}=3)$. Further, patients who were admitted later than $24 \mathrm{~h}$ after symptom 
onset $(\mathrm{n}=28)$ or did not receive an MRI scan within $24 \mathrm{~h}$ after symptom onset $(\mathrm{n}=31)$ or had an MRI scan of poor quality $(\mathrm{n}=11)$ were excluded. Thus, a total of $172 \mathrm{pa}-$ tients met the inclusion criteria.

Of these, PDR was present in 88 patients (51.2\%). The median (interquartile range) PDR volume was $1.1(0.2-$ 4.2) $\mathrm{ml}$, representing $8.6(2.6-17.4) \%$ of total PHE. The median time between symptom onset and MRI scan was $3.0(1.5-9.0) \mathrm{h}$. The clinical, laboratory and imaging characteristics of the patients are listed in table 1. Representative MRI scans of patients with and without PDR are shown in figure 1.

In univariate analyses, patients with PDR had larger hematomas $(53.9(25.4-101.8) \mathrm{ml}$ vs. $9.8(3.6-17.2) \mathrm{ml}$, $\mathrm{p}<0.001)$, higher PHE volumes $(13.8(6.3-27.5) \mathrm{ml}$ vs. 3.8 (1.6-6.7) $\mathrm{ml}, \mathrm{p}<0.001)$, more often lobar hematoma (56.8 vs. $19.0 \%, \mathrm{p}<0.001)$ and possible or probable CAA (50.0 vs. $16.6 \%, \mathrm{p}<0.001)$, higher levels of $\mathrm{C}$-reactive protein (2.52 (1.36-9.58) mg/lvs. 2.06 (1.14-4.08) mg/l, $\mathrm{p}=0.039)$, a lower frequency of diabetes mellitus ( 8.0 vs. $19.0 \%, \mathrm{p}=$ $0.033)$ and a lower systolic blood pressure (160 (141-190) $\mathrm{mm} \mathrm{Hg}$ vs. 180 (155-200) $\mathrm{mm} \mathrm{Hg,} \mathrm{p} \mathrm{=} \mathrm{0.046),} \mathrm{diastolic}$ blood pressure (85 (70-99) mm Hg vs. 93 (85-109) mm $\mathrm{Hg}, \mathrm{p}=0.029)$ and mean arterial blood pressure (111 (97128) $\mathrm{mm} \mathrm{Hg}$ vs. 121 (105-138) mm Hg, p = 0.030; table 1).

In a multivariate logistic regression analysis (adjusted for age, time to MRI, and the above mentioned predictors), PHE volume (OR 1.780, 95\% CI 1.248-2.539, $\mathrm{p}=$ $0.001)$ and diastolic blood pressure (DBP) on admission (OR 0.899, 95\% CI 0.823-0.982, $\mathrm{p}=0.018$ ) were the sole independent predictors of PDR (table 1). However, univariate linear regression revealed a high correlation of hematoma volume and PHE volume $(\beta=0.554, \mathrm{p}<0.001)$, indicating multicollinearity, possibly compromising the results for individual predictors in the above multivariate logistic regression. Thus, multiple linear regression analyses with PDR volume as dependent variable were conducted to verify these results and to account for possible interactions between independent variables (online suppl. tables 1 and 2; for all online suppl. material, see www. karger.com/doi/10.1159/000446549).

Hematoma volume was a significant predictor of PDR volume unless controlled for PHE volume. As hematoma volume was significantly correlated with PHE volume, this indicates that hematoma volume is a predictor of both PDR and PHE. Further, the ratio of PDR volume to PHE volume was not correlated with hematoma volume $(\beta=0.199, \mathrm{p}=0.061)$.

Lobar hematoma location was a significant predictor of PDR volume unless controlled for both hematoma vol- ume and PHE volume. As lobar hematoma location was correlated with hematoma volume $(\beta=0.310, \mathrm{p}<0.001)$ and PHE volume $(\beta=0.327, p=0.001)$, this may indicate that the effect of lobar hematoma location on PDR volume is mediated by both hematoma volume and PHE volume.

DBP was a significant predictor of PDR volume only when controlled for hematoma volume. As DBP was not significantly correlated with PDR volume $(\beta=-0.198, \mathrm{p}=$ $0.079)$ and hematoma volume $(\beta=0.310, p=0.930)$, this may suggest that the effect of DBP on PDR volume is moderated by hematoma volume. No significant interaction between DBP and hematoma location was observed. Possible and probable CAA effects were completely mediated by hematoma location and were not included in the further analysis.

Further, hematoma volume and PHE volume were significantly negatively correlated with ADC values of PDR $(\beta=-0.215, p=0.045$ for hematoma volume and $\beta=$ $-0.267, p=0.012$ for PHE volume, respectively) in univariate linear regression, but not in multivariate linear regression.

PDR was significantly more often located in the perihematomal zone between hematoma and ventricular system $(\mathrm{n}=74,84.0 \%, \mathrm{p}<0.001)$ than in the perihematomal zone between hematoma and adjacent cortex $(\mathrm{n}=7$, $8.0 \%)$ or on both sides $(n=7,8.0 \%)$. No significant difference was found for the location of PDR in the superior or inferior half of the perihematomal zone (superior ( $\mathrm{n}=$ $31), 35.2 \%$; inferior $(n=20), 22.7 \%$, both $(n=37), 42.0 \%$; $\mathrm{p}=0.079$ ). Information on clinical outcome was available in 169 patients (98.3\%). Thirty-six patients survived the initial hospitalization without severe disability $(21.3 \%$; $\mathrm{mRS} \leq 3$ ), 93 patients survived with moderately severe to severe disability ( $55.0 \%$; mRS 4 or 5 ), whereas 40 patients died during the initial hospitalization (23.7\%; mRS 6).

Patients who died during the initial hospitalization were significantly older $(80(72-84)$ years vs. 75 (64-80) years, $\mathrm{p}=0.003)$, were more often on anticoagulants (32.5 vs. $14.0 \%, \mathrm{p}=0.008)$ and had higher serum levels of glucose (8.22 (7.14-9.85) $\mathrm{mmol} / \mathrm{l}$ vs. $6.86(5.36-7.98) \mathrm{mmol} / \mathrm{l}, \mathrm{p}=$ $0.005)$ and CRP (3.39 (1.39-16.69) mg/l vs. 2.22 (1.204.42) $\mathrm{mg} / \mathrm{l}, \mathrm{p}=0.008)$, respectively. Regarding imaging parameters, patients who died during the initial hospitalization presented significantly more often with PDR (75.0 vs. $43.4 \%, \mathrm{p}<0.001)$, higher volumes of PDR $(2.2(0.7-7.0) \mathrm{ml}$ vs. $0.5(0.2-2.6) \mathrm{ml}, \mathrm{p}=0.001)$, higher hematoma volume (106.8 (43.2-146.5) $\mathrm{ml}$ vs. $14.0(4.6-34.0) \mathrm{ml}, \mathrm{p}<0.001)$, higher PHE volumes (17.0 (7.4-38.1) $\mathrm{ml}$ vs. 5.5 (2.6-11.8) $\mathrm{ml}, \mathrm{p}<0.001)$, higher frequency of ventricular extension of 
Table 1. Clinical, laboratory and imaging characteristics of patients with and without PDR

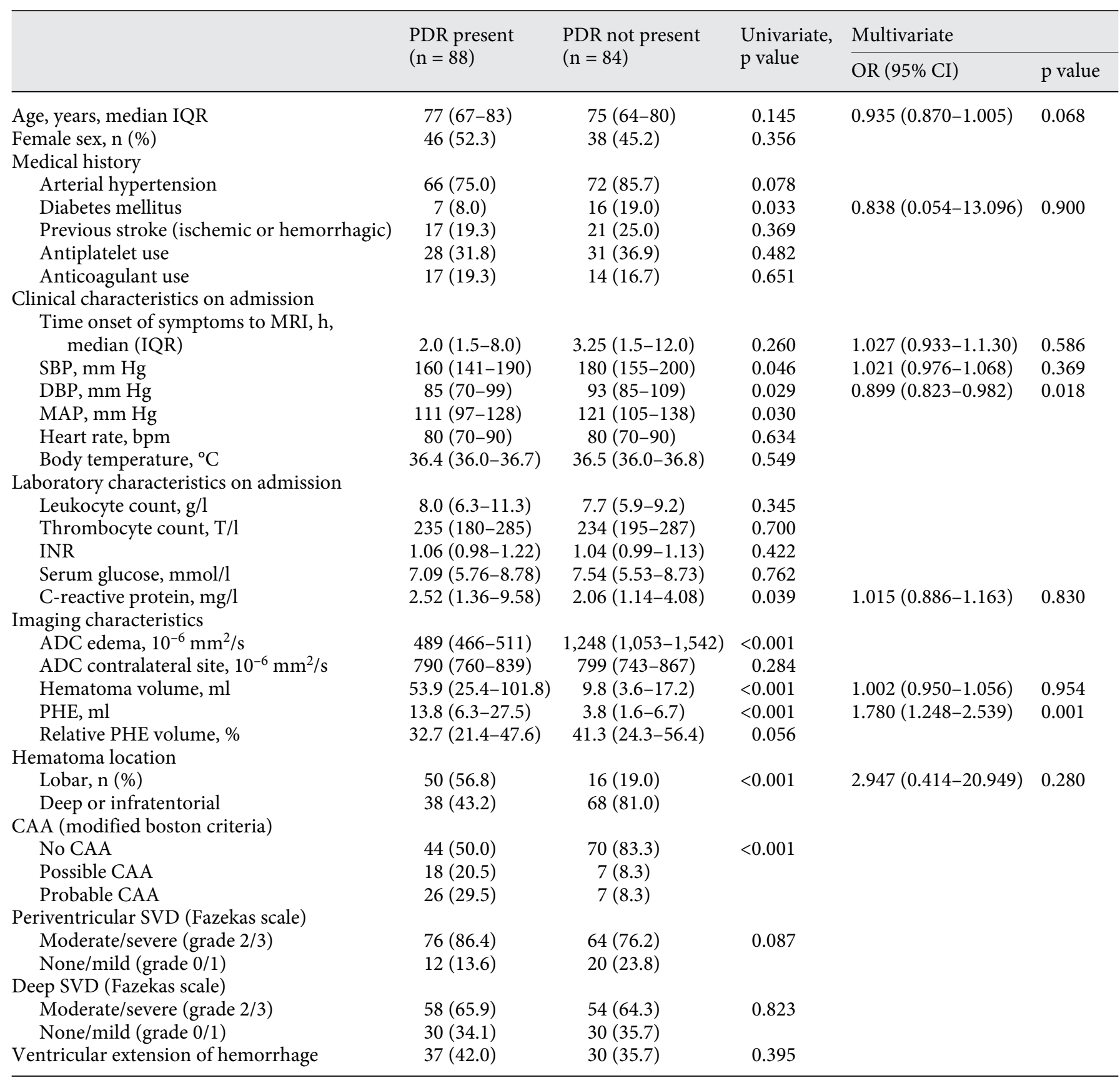

$\mathrm{IQR}=$ Interquartile range; $\mathrm{SBP}=$ systolic blood pressure; $\mathrm{MAP}=$ mean arterial blood pressure; $\mathrm{INR}=$ international normalized ratio; $\mathrm{SVD}=$ small vessel disease.

the hemorrhage $(72.5$ vs. $27.9 \%, \mathrm{p}<0.001)$ and more often with lobar hematoma ( 52.5 vs. $34.9 \%, \mathrm{p}=0.046$ ).

In a multivariate logistic regression model (adjusted for the above-mentioned predictors and time to MRI), age (OR 1.142, 95\% CI 1.022-1.276, $\mathrm{p}=0.019$ ), hemato- ma volume (OR 1.043, 95\% CI 1.005-1.083, $\mathrm{p}=0.027$ ) and ventricular extension (OR 27.250, 95\% CI 1.497495.927, $\mathrm{p}=0.026$ ) were the sole independent predictors of mortality. PDR and PHE volumes were no independent predictors of mortality (table 2 ). 


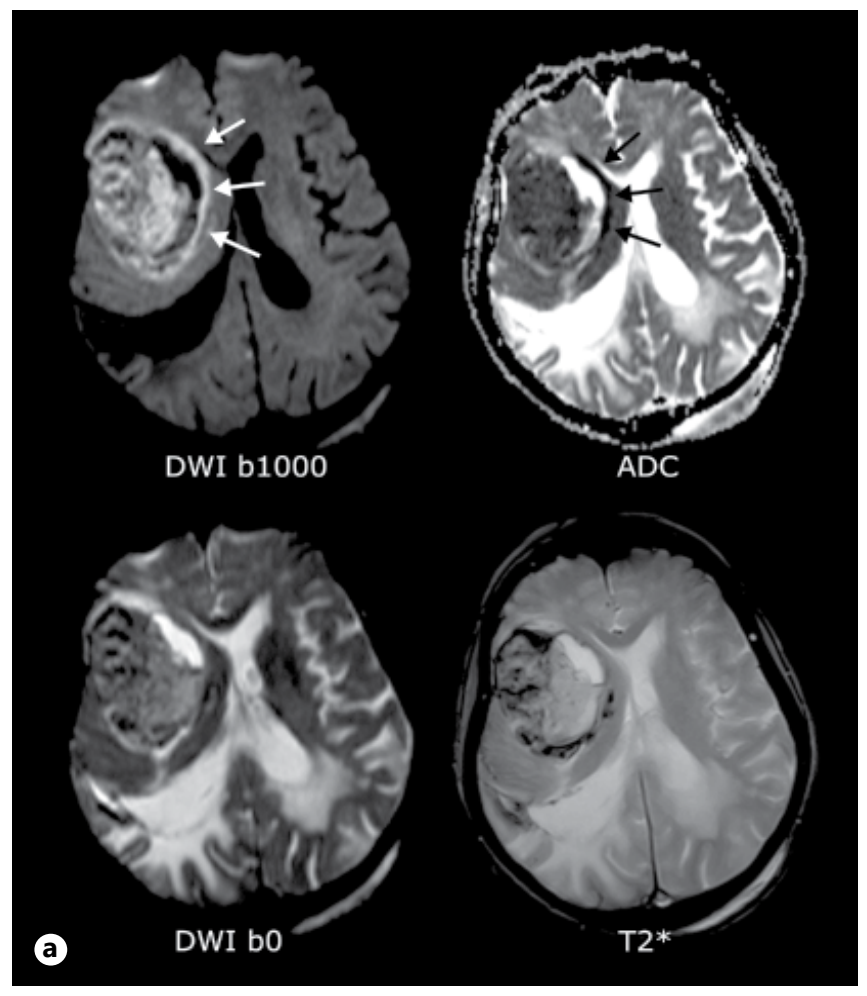

Fig. 1. MRI characteristics of the perihematomal zone in patients with ICH. a Lobar hematoma with PDR as indicated by hyperintense signal of the perihematomal zone on DWI b1000 map and hypointense signal on ADC map (arrows). DWI b0 and T2* maps

The predictors of death and severe disability (mRS 4-6) vs. survival without moderately severe to severe disability ( $\mathrm{mRS} \leq 3)$ are shown in online supplemental table 3. Briefly, hematoma volume (OR 1.075, 95\% CI $1.004-1.151, \mathrm{p}=0.039)$ and ventricular extension (OR $5.015,95 \%$ CI $1.456-17.274, \mathrm{p}=0.011$ ) were identified as the sole independent predictors of death and severe disability in a multivariate logistic regression model.

Additionally, mRS shift analysis was conducted by multiple ordinal regression analysis (online suppl. table 4). Higher mRS scores, indicating worse clinical outcome, were predicted by higher age (OR 1.047, 95\% CI $1.021-1.074, \mathrm{p}<0.001$ ), larger hematoma (OR 1.033, 95\% CI 1.017-1.049, $\mathrm{p}<0.001)$, ventricular extension of hemorrhage (OR 4.286, 95\% CI 2.150-8.543, p < 0.001) and shorter latency between symptom onset and MRI (OR 0.956, 95\% CI 0.922-0.991, $\mathrm{p}=0.014)$. PDR and PHE did not independently predict a shift toward worse outcome.

The estimated power to detect an independent association of PDR and clinical outcome in the multivariate regression analyses was 75,65 and $80 \%$ with mortality,

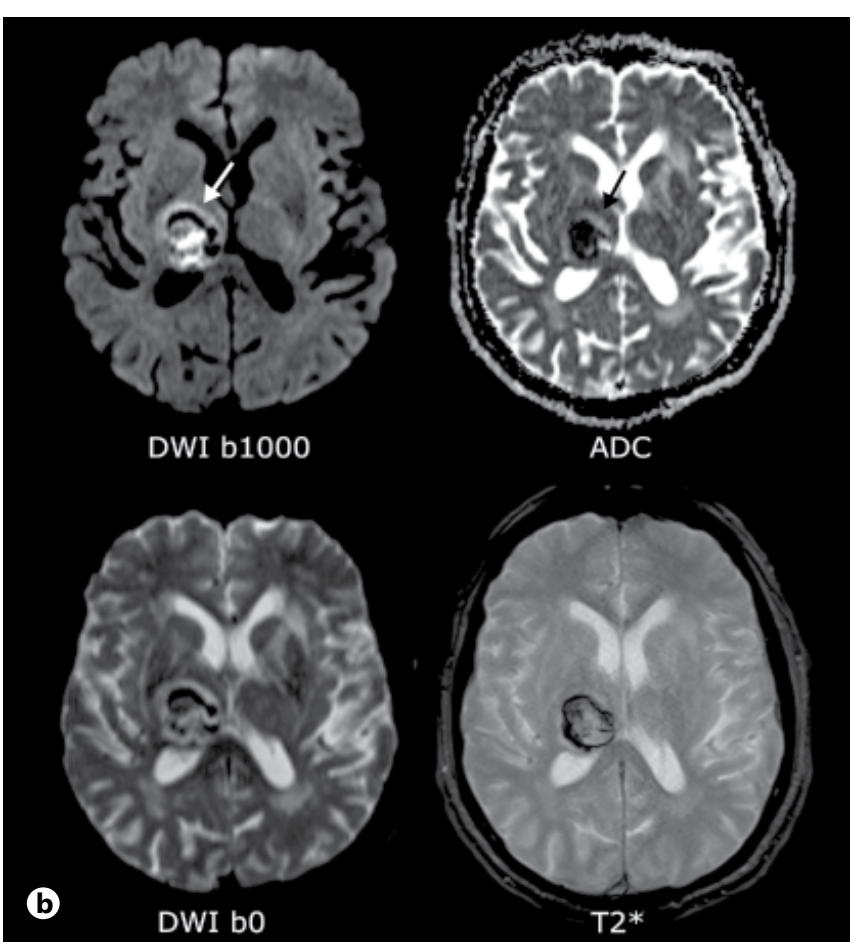

are shown for anatomical reference. b Deep thalamic hematoma with perihematomal vasogenic edema as indicated by hyperintense signal on DWI b1000 and ADC maps (arrows).

mortality/disability and mRS shift, respectively, as dependent variable (with $\alpha=0.05$ and correction for covariates).

\section{Discussion}

In our retrospective cohort study of 172 patients with primary $\mathrm{ICH}$, areas of restricted diffusion within the perihematomal zone (PDR) were observed in more than half of the patients within $24 \mathrm{~h}$ of symptom onset (51.2\%). Hematoma volume, PHE volume, lobar hematoma location and low DBP at admission were identified as predictors of PDR. Hematoma volume was, however, identified as the key predictor, being highly correlated with PHE volume, partially mediating the effect of lobar hematoma location and moderating the effect of DBP.

Previous studies reported PDR in $10-70 \%$ of all cases; however, most of these studies were limited by small sample sizes [9-13]. The temporal pattern of ADC properties of the perihematomal zone was previously shown to be 
Table 2. Clinical, laboratory and imaging characteristics of patients who died or survived the initial hospitalization

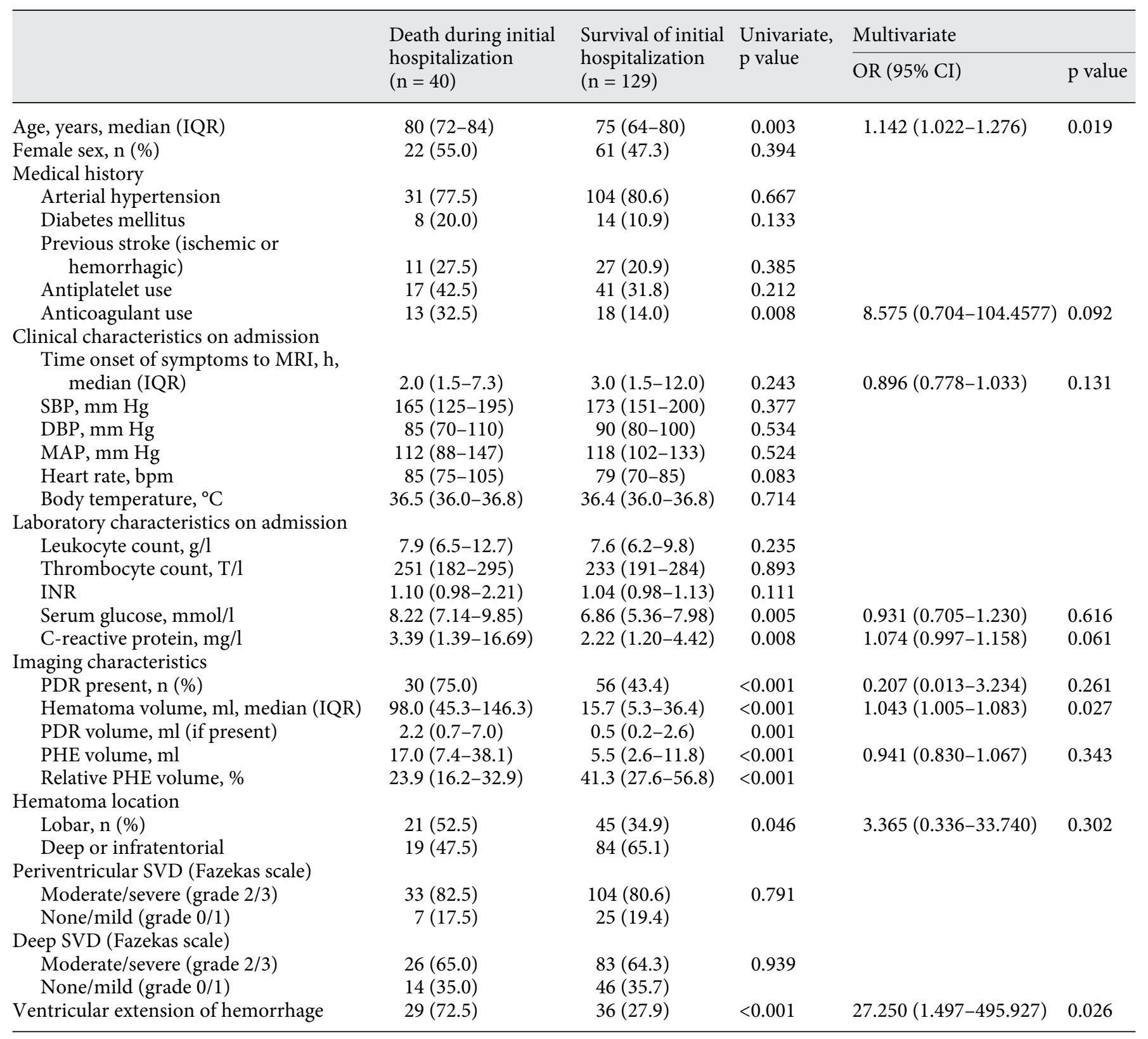

$\mathrm{IQR}=$ Interquartile range; $\mathrm{SBP}=$ systolic blood pressure; $\mathrm{MAP}=$ mean arterial blood pressure; $\mathrm{INR}=$ international normalized ratio; $\mathrm{SVD}=$ small vessel disease.

highly variable. In particular, one study reported a decrease in frequency of PDR from $45 \%$ on day 1 after symptom onset to $32 \%$ on day 7 [11]. This observation may account for the relatively high frequency of PDR in our sample compared to previous studies, since only patients with MRI obtained within $24 \mathrm{~h}$ after symptom onset were included in our sample, whereas previous studies included patients with MRI scans up to 14 days after symptom onset [13].

The pathophysiology of PDR is unclear. As water diffusion in tissues is mainly limited by cellular membranes, changes in the microstructure, such as cellular swelling in cytotoxic edema or mechanical compression of white matter tracts may lead to restricted water diffusion [24]. 
In the perihematomal zone, possible mechanisms accounting for PDR include ischemia with subsequent cytotoxic edema, mechanical compromise due to space-occupying hematoma and toxic or inflammatory effects related to components of the hematoma (e.g. thrombin or matrix metalloproteinases) [25]. MRI and CT perfusion studies demonstrated delayed perfusion of the perihematomal zone within the first $24 \mathrm{~h}$ after ICH with a normalization of perfusion within the first few days [26-28]. This delay of perfusion was correlated with hematoma volume [26]. However, the degree of perfusion delay did not reach the threshold for true ischemia, as observed in ischemic stroke [26-28]. PET and CT perfusion studies on the metabolism of the perihematomal region demonstrated reduced oxygen extraction fraction and cerebral metabolic rate of oxygen, indicative of reduced metabolic demands rather than ischemia $[29,30]$. Considering these observations, true ischemic injury in the perihematomal zone is thought to be unlikely.

Although restricted diffusion in DWI-MRI imaging is suggestive of cytotoxic edema secondary to ischemia, compression and displacement of white matter tracts may also cause restricted diffusion, as observed in PDR. Our study identified hematoma volume as the key predictor of PDR and also PHE volume, which is in line with previous studies $[5,10,13,31]$. This possibly suggests mechanical compression as a causal factor underlying PDR. This assumption is supported by the observation that PDR was most often located in the white matter in between the hematoma and the ventricular system, where mechanical displacement may be more pronounced than in white matter in between the hematoma and the cortex. Additionally, higher hematoma volumes were associated with more profound diffusion restriction, as indicated by lower ADC values, providing another link between hematoma volume and PDR.

Although PHE volumes were associated with higher PDR volumes, the ratio of PDR volume to PHE volume was constant across different hematoma volumes. This may suggest that PDR and PHE are not pathophysiologically distinct entities, but rather represent parts of the same spectrum of tissue responses to ICH. Although low DBP was an independent predictor of PDR, this effect was dependent on hematoma volume. Likewise, lobar hematoma location was predictive of PDR, but higher hematoma volumes and higher PHE volumes mediated this effect.

PDR has been repeatedly suggested as a prognostic and therapeutic target in ICH [9-11]. In our cohort, well known risk factors, that is, hematoma volume, ventricular extension of hemorrhage and higher age were inde- pendent predictors of mortality and severe disability at hospital discharge [3]. A shorter latency between symptom onset and initial MRI scan was an additional independent predictor of a worse functional outcome. Although PDR within $24 \mathrm{~h}$ of symptom onset was associated with worse clinical outcome in our cohort, PDR was not an independent predictor of death and severe disability. Congruently with this, PHE was no independent predictor of worse clinical outcome. As hematoma volume was the main predictor of PDR, our data do not support a role of early PDR in determining clinical outcome after ICH independent of hematoma volume. Similar observations were made for PHE in larger hematoma [5, 32]. The fact that PDR volume was relatively small compared to hematoma volume might explain why no independent impact of PDR on clinical outcome was observed.

The retrospective and single-center design of our study bears known limitations. As PDR was evaluated only at a single early point in time, we could not account for chronological fluctuations of PDR. Blood pressure measures were analyzed for the time of admission only because data were incomplete at later time points (due to early death or transfer of patients). mRS scores were determined retrospectively by chart review, which may have led to misclassification of patients, in particular with a score of 4 or 5. Additionally, mRS scores at hospital discharge may not accurately reflect long-term outcome. Regarding mortality, we could not correct for do-not-resuscitate orders or limitation of care decisions.

\section{Conclusions}

PDR is common after primary ICH within $24 \mathrm{~h}$ of symptom onset. Multivariate analyses identified hematoma volume as key predictor of PDR, whereas the volume of PHE, lobar hematoma location and low DBP at admission were additional predictors. This may suggest that mechanical compromise is a main factor in the pathogenesis of PDR. Although PDR was associated with mortality and severe disability, this effect was confounded by established risk factors. These results do not support a role of early PDR as prognostic factor after ICH independent of hematoma volume.

\section{Acknowledgments}

The authors thank J. Kudliczka for IT assistance in identifying patients via the hospital information system. 


\section{Sources of Funding}

None.

\section{References}

1 Broderick JP, Brott TG, Duldner JE, Tomsick T, Huster G: Volume of intracerebral hemorrhage. A powerful and easy-to-use predictor of 30-day mortality. Stroke 1993;24:987-993.

2 Balami JS, Buchan AM: Complications of intracerebral haemorrhage. Lancet Neurol 2012;11:101-118.

3 Hemphill JC 3rd, Bonovich DC, Besmertis L, Manley GT, Johnston SC: The ICH score: a simple, reliable grading scale for intracerebral hemorrhage. Stroke 2001;32:891-897.

4 Yang J, Arima H, Wu G, Heeley E, Delcourt C, Zhou J, et al: Prognostic significance of perihematomal edema in acute intracerebral hemorrhage: pooled analysis from the intensive blood pressure reduction in acute cerebral hemorrhage trial studies. Stroke 2015;46: 1009-1013.

5 Murthy SB, Moradiya Y, Dawson J, Lees KR, Hanley DF, Ziai WC, et al: Perihematomal edema and functional outcomes in intracerebral hemorrhage: influence of hematoma volume and location. Stroke 2015;46:3088-3092.

6 Gebel JM Jr, Jauch EC, Brott TG, Khoury J, Sauerbeck L, Salisbury S, et al: Relative edema volume is a predictor of outcome in patients with hyperacute spontaneous intracerebral hemorrhage. Stroke 2002;33:2636-2641.

7 Volbers B, Willfarth W, Kuramatsu JB, Struffert T, Dörfler A, Huttner HB, et al: Impact of perihemorrhagic edema on short-term outcome after intracerebral hemorrhage. Neurocrit Care 2015, Epub ahead of print.

8 Huisman TA: Diffusion-weighted imaging: basic concepts and application in cerebral stroke and head trauma. Eur Radiol 2003;13: 2283-2297.

9 Fainardi E, Borrelli M, Saletti A, Sarubbo S, Roversi G, Bernardoni A, et al: Temporal changes in perihematomal apparent diffusion coefficient values during the transition from acute to subacute phases in patients with spontaneous intracerebral hemorrhage. Neuroradiology 2013;55:145-156.

10 Kidwell CS, Saver JL, Mattiello J, Warach S, Liebeskind DS, Starkman S, et al: Diffusionperfusion MR evaluation of perihematomal injury in hyperacute intracerebral hemorrhage. Neurology 2001;57:1611-1617.
11 Li N, Worthmann H, Heeren M, Schuppner $\mathrm{R}$, Deb M, Tryc AB, et al: Temporal pattern of cytotoxic edema in the perihematomal region after intracerebral hemorrhage: a serial magnetic resonance imaging study. Stroke 2013; 44:1144-1146.

12 Olivot JM, Mlynash M, Kleinman JT, Straka $\mathrm{M}$, Venkatasubramanian C, Bammer R, et al: MRI profile of the perihematomal region in acute intracerebral hemorrhage. Stroke 2010; 41:2681-2683.

13 Gioia LC, Kate M, Choi V, Sivakumar L, Jeerakathil T, Kosior J, et al: Ischemia in intracerebral hemorrhage is associated with leukoaraiosis and hematoma volume, not blood pressure reduction. Stroke 2015;46:15411547.

14 van Swieten JC, Koudstaal PJ, Visser MC Schouten HJ, van Gijn J: Interobserver agreement for the assessment of handicap in stroke patients. Stroke 1988;19:604-607.

15 Schindelin J, Arganda-Carreras I, Frise E, Kaynig V, Longair M, Pietzsch T, et al: Fiji: an open-source platform for biological-image analysis. Nat Methods 2012;9:676-682.

16 Uluğ AM, Beauchamp N Jr, Bryan RN, van Zijl PC: Absolute quantitation of diffusion constants in human stroke. Stroke 1997;28: 483-490.

17 Bastin ME, Rana AK, Wardlaw JM, Armitage PA, Keir SL: A study of the apparent diffusion coefficient of grey and white matter in human ischaemic stroke. Neuroreport 2000;11:28672874.

18 Pierpaoli C, Alger JR, Righini A, Mattiello J, Dickerson R, Des Pres D, et al: High temporal resolution diffusion MRI of global cerebral ischemia and reperfusion. J Cereb Blood Flow Metab 1996;16:892-905.

19 Venkatasubramanian C, Mlynash M, FinleyCaulfield A, Eyngorn I, Kalimuthu R, Snider RW, et al: Natural history of perihematomal edema after intracerebral hemorrhage measured by serial magnetic resonance imaging. Stroke 2011;42:73-80.

20 Fazekas F, Chawluk JB, Alavi A, Hurtig HI, Zimmerman RA: MR signal abnormalities at $1.5 \mathrm{~T}$ in Alzheimer's dementia and normal aging. AJR Am J Roentgenol 1987;149:351-356.

21 Knudsen KA, Rosand J, Karluk D, Greenberg SM: Clinical diagnosis of cerebral amyloid angiopathy: validation of the Boston criteria. Neurology 2001;56:537-539.
22 Linn J, Halpin A, Demaerel P, Ruhland J, Giese AD, Dichgans M, et al: Prevalence of superficial siderosis in patients with cerebral amyloid angiopathy. Neurology 2010;74: 1346-1350.

23 Faul F, Erdfelder E, Buchner A, Lang AG: Statistical power analyses using $G^{*}$ Power 3.1: tests for correlation and regression analyses. Behav Res Methods 2009;41:1149-1160.

24 Le Bihan D, Johansen-Berg H: Diffusion MRI at 25: exploring brain tissue structure and function. NeuroImage 2012;61:324-341.

25 Xi G, Keep RF, Hoff JT: Mechanisms of brain injury after intracerebral haemorrhage. Lancet Neurol 2006;5:53-63.

26 Butcher KS, Baird T, MacGregor L, Desmond $\mathrm{P}$, Tress B, Davis S: Perihematomal edema in primary intracerebral hemorrhage is plasma derived. Stroke 2004;35:1879-1885.

27 Schellinger PD, Fiebach JB, Hoffmann K, Becker K, Orakcioglu B, Kollmar R, et al: Stroke MRI in intracerebral hemorrhage: is there a perihemorrhagic penumbra? Stroke 2003;34:1674-1679.

28 Pascual AM, López-Mut JV, Benlloch V, Chamarro R, Soler J, Láinez MJ: Perfusionweighted magnetic resonance imaging in acute intracerebral hemorrhage at baseline and during the 1st and 2nd week: a longitudinal study. Cerebrovasc Dis 2007;23:6-13.

29 Zazulia AR, Diringer MN, Videen TO, Adams RE, Yundt K, Aiyagari V, et al: Hypoperfusion without ischemia surrounding acute intracerebral hemorrhage. J Cereb Blood Flow Metab 2001;21:804-810.

30 Kate MP, Hansen MB, Mouridsen K, Østergaard L, Choi V, Gould BE, et al: Blood pressure reduction does not reduce perihematoma oxygenation: a CT perfusion study. J Cereb Blood Flow Metab 2014;34:81-86.

31 Staykov D, Wagner I, Volbers B, Hauer EM, Doerfler A, Schwab S, et al: Natural course of perihemorrhagic edema after intracerebral hemorrhage. Stroke 2011;42:2625-2629.

32 Arima H, Wang JG, Huang Y, Heeley E, Skulina C, Parsons MW, et al: Significance of perihematomal edema in acute intracerebral hemorrhage: the INTERACT trial. Neurology 2009;73:1963-1968. 